\title{
Robust technique for spectroscopic plasma analysis with application in real-time arc welding quality monitoring
}

\section{J. Mirapeix \\ A. Cobo \\ O. M. Conde}

Universidad de Cantabria

Grupo de Ingeniería Fotónica

Department TEISA

Avda. Los Castros s/n

39005 Santander, Spain

E-mail: mirapeixjm @unican.es

\section{Jaúregui}

University of Southampton

Optoelectronics Research Centre

SO17 1BJ, United Kingdom

J. M. López-Higuera, MEMBER SPIE

Universidad de Cantabria

Grupo de Ingeniería Fotónica

Department TEISA

Avda. Los Castros s/n

39005 Santander, Spain

\begin{abstract}
A new real-time implementation of the Boltzmann-plot technique for optical spectroscopic analysis of thermal plasmas is presented. Applications are found in the monitoring of industrial processes in which plasma emission naturally occurs, such as arc and laser welding. Experimental results show the feasibility of using the Boltzmann-plot technique for real-time calculation of the electronic temperature of the plasma, a parameter that has been correlated with common defects found in the weld seam. The foundations of the proposed technique, its implementation, and its capability for the on-line detection of defects in tungsten inert gas (TIG) welding of steel parts are discussed. (๑) 2006 Society of Photo-Optical Instrumentation Engineers. [DOI: 10.1117/1.2336416]
\end{abstract}

Subject terms: spectroscopy; plasma emission; arc welding; real-time processing.

Paper 050759R received Sep. 22, 2005; revised manuscript received Jan. 27, 2006; accepted for publication Jan. 30, 2006; published online Aug. 22, 2006.

\section{Introduction}

Quality assurance of the welding process is of major importance in some critical applications, such as those within the aerospace sector, in which extensive nondestructive testing (NDT) procedures are routinely performed to every welded part. For these applications, the early detection of welding defects by means of an on-line quality monitoring system could avoid expensive NDT of early-identified defective weld seams.

Several monitoring principles have been demonstrated for this particular process, such as the measurement of the charge voltage induced on the welding nozzle, ${ }^{1}$ acoustic emissions, ${ }^{2}$ or imaging in different spectral bands (including infrared thermography ${ }^{3}$ ). It is worth noting that some welding methods, like arc welding or laser welding in the so-called "keyhole" regime, exhibit an inherent plasma emission in the ultraviolet (UV) and visible bands. The analysis of the emitted light offers relevant information about the welding process itself and, ultimately, about the quality of the resulting weld. ${ }^{4}$

These optical approaches to quality monitoring exhibit some advantages over electrical or acoustic methods, one of the most important being their immunity to the strong electromagnetic interferences (EMI) typically found in these processes, especially if the light is collected far from the welding torch. This is usually not a problem due to the high intensity from the thermal plasma, and setups with remote photodiodes ${ }^{5}$ and optical fiber capturing ${ }^{6}$ have been

0091-3286/2006/\$22.00 @ 2006 SPIE demonstrated. Even the focusing optics of a welding laser system can be used to back-propagate the plasma emission from the welding spot. ${ }^{7}$

Another advantage of the optical methods refers to the rich information they can offer of the welding process itself. In particular, the spectroscopic analysis of the plasma emission reveals the characteristic and complex pattern of multiple emission peaks due to the multiple atomic species (at different degrees of ionization) contributing to the plasma. The processing of the collected spectrum involves typically the estimation of the amplitude and width of those peaks, along with the identification of the associate atomic species. From this data, relevant information of the plasma composition can be derived, which in turn can be correlated with some properties of the resulting welding seam. ${ }^{8}$

The most widely employed technique when a plasma is spectroscopically analyzed is the calculation of its electronic temperature, as it has been demonstrated that there is a relationship between this parameter and the resulting quality of the weld seam. ${ }^{8-10}$ The reliable acquisition and subsequent analysis of the electronic temperature of the plasma $T_{e}$ allows the identification of defects that can appear in the weld seam as a result of a wide variety of causes: intensity fluctuations, shielding gas flux variations, irregularities in the width of the welded materials, and many others, not to mention those defects generated by human operators.

Although the Boltzmann-plot method permits a precise calculation of the plasma electronic temperature, ${ }^{4,11}$ the simplification that estimates $T_{e}$ from a single pair of emis- 
sion lines of a given species is usually assumed valid. Computational cost is the main constriction when this solution is adopted, especially if real-time operation is required, as it is desirable when a sensor system is designed not only to detect possible defects, but also to actuate over the process to assure a result with a specific quality.

In a previous work, ${ }^{12}$ a real-time monitoring principle for the analysis of thermal plasmas was proposed. With this technique, the central peak wavelength estimation and, hence the spectral line identification, was performed by using the lineal phase operator (LPO) subpixel technique. ${ }^{13}$ By substituting the traditional solutions used for the spectral lines characterization, like the Levenberg-Marquardt technique, this approach allows the real-time calculation of the electronic temperature of the plasma $T_{e}$ using a single pair of spectral lines. While the use of the LPO algorithm allows a more accurate estimation of the central wavelength associated to each spectral line, a cubic-spline interpolation of the noisy data is performed to obtain the peak intensities.

In this work, we propose to extend the previously described technique to multiple spectral lines using the Boltzmann-plot method for the $T_{e}$ calculation. The results show that a robust estimation of $T_{e}$ is obtained, while still being able to operate in real time. The proposed technique has been applied to on-line defect detection in arc welding processes. The correlation between the calculated $T_{e}$ and defects produced in the weld seams are analyzed, along with performance comparisons with the previously reported method based on single pairs of spectral lines. The experimental results of tungsten inert gas (TIG) welds performed on steel plates show that the proposed technique is able to detect, in real-time, common defects such as oxidation due to insufficient flux of shielding gas, or lack (or excess) of penetration of the weld seam.

\section{Theoretical Background}

The detection of defects in the weld seam by means of spectroscopic analysis implies the necessity of finding a correlation between the weld quality and its defects and the electronic temperature of the plasma. This parameter can be obtained from the relative intensities of some emission lines belonging to a particular atomic species within those participating in the plasma. ${ }^{4}$ If the electron density is high enough so that:

$N_{e} \geqslant 1.6 \times 10^{12} T_{e}^{1 / 2}(\Delta E)^{3}$,

(where $N_{e}$ is the electronic density, $T_{e}$ is the electronic temperature, and $\Delta E$ is the largest energy gap in the atomic energy level system), then it can be assumed that the plasma is in local thermal equilibrium (LTE), which implies that the various particles within the plasma have Maxwellian energy distributions and collisional processes exceed radiative ones.

When LTE is satisfied, the population of an excited level can be determined with the Boltzmann equation:

$N_{m}=\frac{N}{Z} g_{m} \exp \left(\frac{-E_{m}}{\kappa T_{e}}\right)$,

where $N$ is the population density of the state $m, Z$ is the partition function, $g_{m}$ is the statistical weight, $E_{m}$ is the

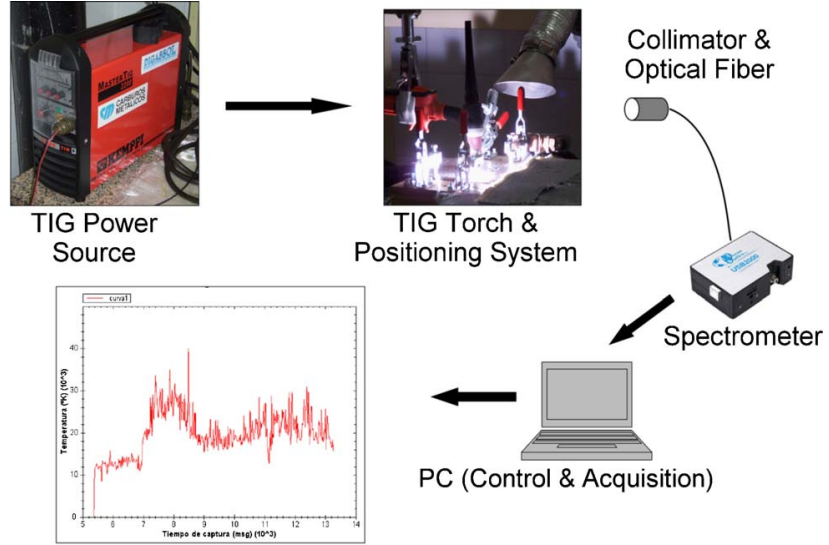

Real-Time Electronic Temperature

Fig. 1 Experimental setup.

excitation energy, and $\kappa$ is the Boltzmann constant.

In optically thin plasmas, the intensity of a given spectral line $I_{m n}$, induced by a transition from level $m$ to level $n$, can be related to the population density of the upper level $N_{m}$ through

$I_{m n}=N_{m} A_{m n} h \gamma_{m n}$,

with $A_{m n}$ being the transition probability, and $h \gamma_{m}$ the energy of such transition.

Combining Eqs. (2) and (3), $T_{e}$ can be obtained from the following expression,

$\ln \left(\frac{I_{m n} \lambda_{m n}}{A_{m n} g_{m}}\right)=\ln \left(\frac{h c N}{Z}\right)-\frac{E_{m}}{k T_{e}}$,

as the plot resulting from using various lines and representing the left-hand side of Eq. (4) versus $E_{m}$ has a slope inversely proportional to $T_{e}$.

A common simplification in the $T_{e}$ calculation process is usually adopted by considering only a pair of spectral lines of a given species. Thus, by knowing the ratio of the relative intensities of those lines and using Eq. (4), $T_{e}$ can be calculated by means of the following expression

$T_{e}=\frac{E_{m}(2)-E_{m}(1)}{k \ln \left[\frac{I(1) A(2) g_{m}(2) \lambda(1)}{I(2) A(1) g_{m}(1) \lambda(2)}\right]}$

From Eq. (5), it is clear that a precise estimation of the central peak wavelength of each considered emission line is needed, especially considering that this value will be used in the identification of the chemical species associated to that line. As was mentioned before, the utilization of the linear phase operator subpixel algorithm will allow an efficient fulfilment of this step. ${ }^{12}$

To check the real-time behavior and performance of this technique, several experiments in the laboratory have been performed. They have been based on TIG welding of steel plates, with common defects intentionally produced. The experimental setup and results are discussed in the next section. 


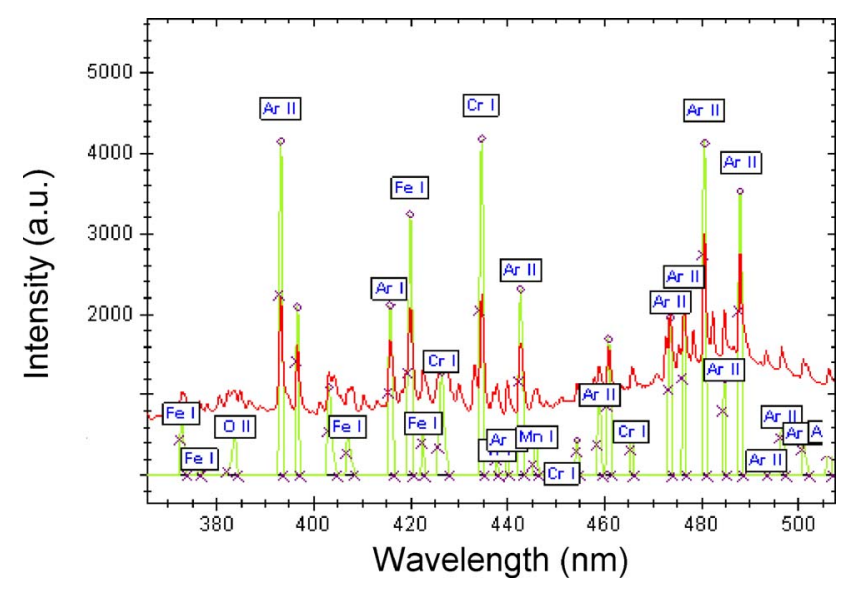

Fig. 2 Spectral lines within the range to be considered.

\section{Experimental Setup and Results}

The experimental setup, designed around a TIG welding system, is schematically described in Fig. 1. The power source of the welding system is the Kemppi Mastertig 2200, which offers output intensities from 5 to 220 A. The motion of the weld system is obtained by keeping the torch fixed and displacing the plates to be welded with a highprecision ( $1 \mu \mathrm{m}$ in both axes) positioning system, formed by the Newport MM4005 controller and two Newport MTM100PP1 linear stages. Argon, with typical flows of $12 \mathrm{~L} / \mathrm{min}$, has been used as a shielding gas. 1-mm-diam tungsten rods and AISI-304 stainless steel plates have been used in our experiments, although the consideration of any other material does not imply the necessity of a previous calibration of the system.

A collimating lens and a solarization-resistant optical fiber (Ocean Optics P400-UV-SR) were respectively used to acquire the light radiation from the plasma and to guide it to the entrance of the spectrometer. A low-cost, 2048 linear charge-coupled device (CCD) spectrometer (Ocean Optics

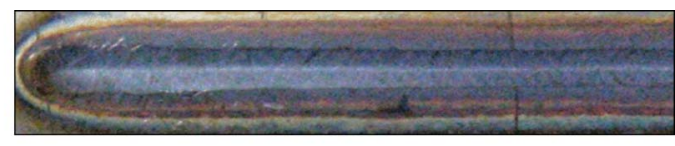

a) Reference Weld Seam

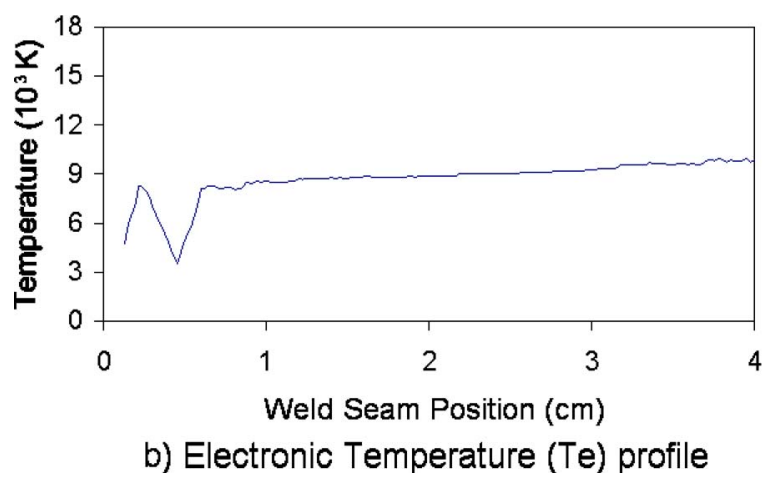

Fig. 3 Example of a reference weld seam without defects.

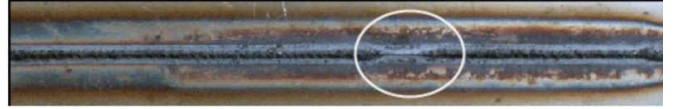

a) Weld Seam (bottom) with defect

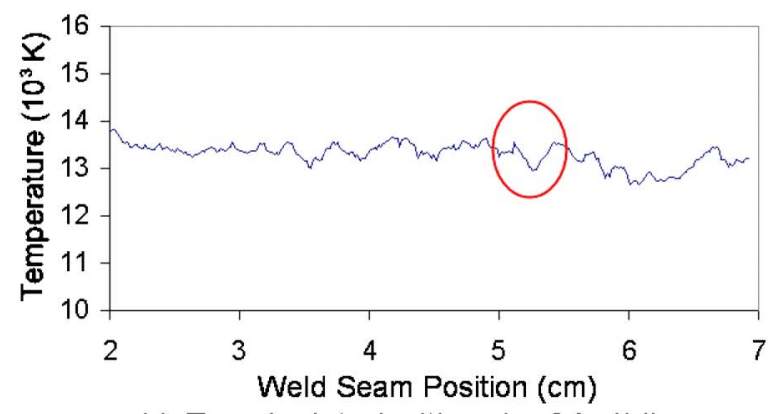

b) Te calculated with pair of Ar II lines

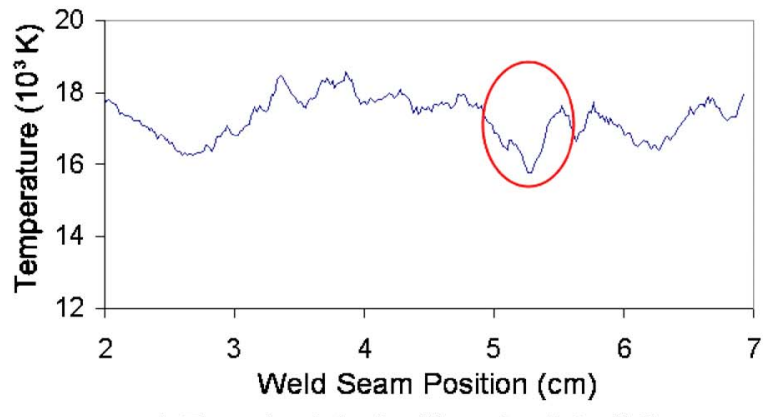

c) Te calculated with pair of Ar II lines

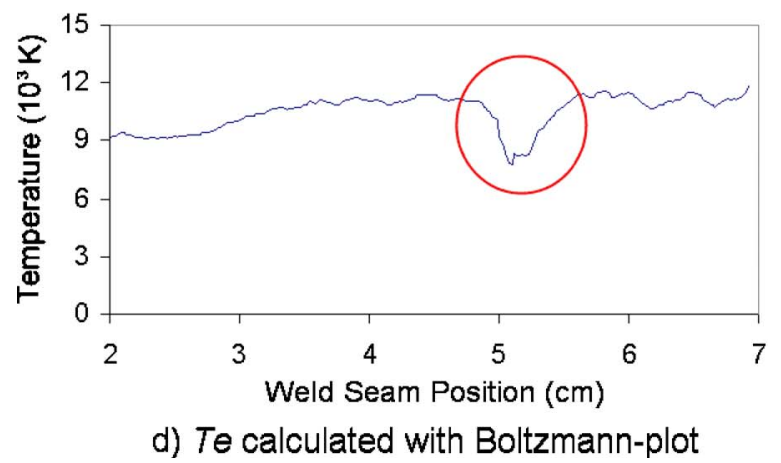

Fig. 4 Detection of weld seam defects with different techniques.

USB2000), with $0.3 \mathrm{~nm}$ of spectral resolution and a spectral range from 195 to $535 \mathrm{~nm}$, was chosen.

Although spectral lines from various species were identified, Ar II and Fe I lines have been used for the estimation of $T_{e}$. Particularly, Ar II emission lines located from 459 to $506 \mathrm{~nm}$, and Fe I lines from 372 to $416 \mathrm{~nm}$, have been systematically used in the Boltzmann-plot technique. In Fig. 2, a typical spectrum obtained from the plasma of an arc weld is presented.

Although the final objective of the proposed system is to detect defects in weld seams, it was considered necessary to previously obtain a reference weld without defects. In Fig. 3 , an example of a weld seam performed with a constant intensity of $42 \mathrm{~A}$ and a constant gas flux of $12 \mathrm{~L} / \mathrm{min}$ is shown. The $T_{e}$ signal depicted in Fig. 3(b) was calculated with four Ar II lines (located at 461, 480, 485, and $488 \mathrm{~nm}$ ) as input data for the Boltzmann-plot process. After an ini- 


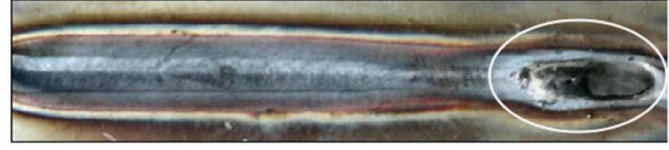

a) Weld Seam (top) with defects

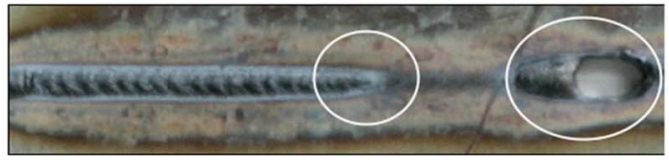

b) Weld Seam (bottom) with defects

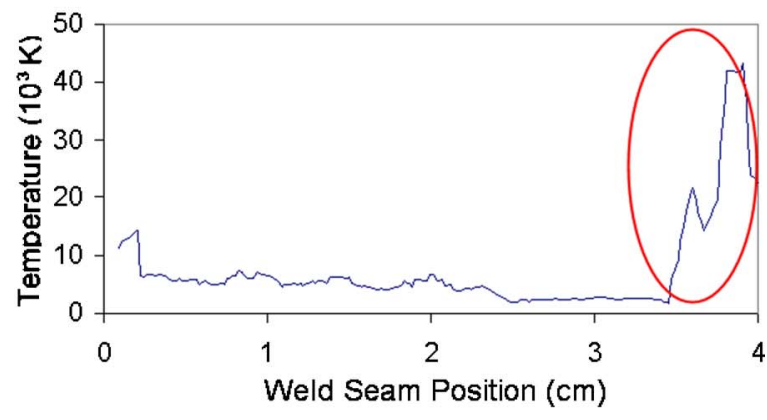

c) Te calculated with pair of $\mathrm{Ar}$ II lines

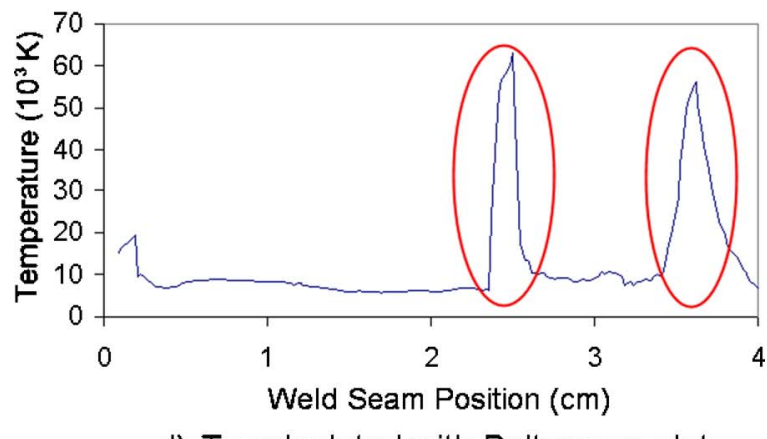

d) Te calculated with Boltzmann-plot

Fig. 5 Detection of weld seam crater.

tial transient state due to the instability at the beginning of the welding process, the $T_{e}$ signal shows no relevant variations, as no visible defects are to be found.

In Fig. 4, a weld seam with a small defect is presented. This defect was caused on a plate with a thickness of $1 \mathrm{~mm}$ by a reduction in the gas flux from $12 \mathrm{~L} / \mathrm{min}$ to approximately $6 \mathrm{~L} / \mathrm{min}$ at $x \approx 5 \mathrm{~cm}$. The resulting weld seam is shown in Fig. 4(a). Figures 4(b) (Ar II lines located at 459 and $488 \mathrm{~nm}$ ) and 4(c) (Ar II lines located at 459 and $485 \mathrm{~nm}$ ) present the result of calculating $T_{e}$ with the classical approach indicated in Eq. (5). The selection of those lines was performed by trying to maximize the difference between their excitation energies $E_{m}$, which leads to a more precise $T_{e}$ determination. ${ }^{14}$ It can be observed in Figs. 4(b) and 4(c) that the defect created by the reduction in the gas flux in the weld seam is identified, but in the first case the change in the $T_{e}$ profile is too small to be detected.

Additionally, the performance of the proposed technique has been checked by using the Boltzmann plot with six Ar II lines (located at 404, 459, 473, 476, 485, and $488 \mathrm{~nm}$ ) to calculate $T_{e}$. As expected, the sensitivity to defects in-

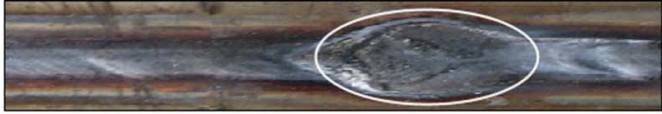

a) Weld Seam (top) with defects

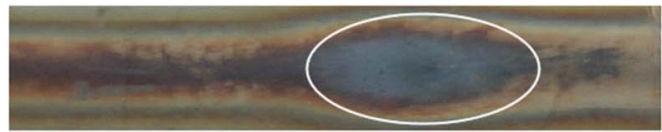

b) Weld Seam (bottom) with defects

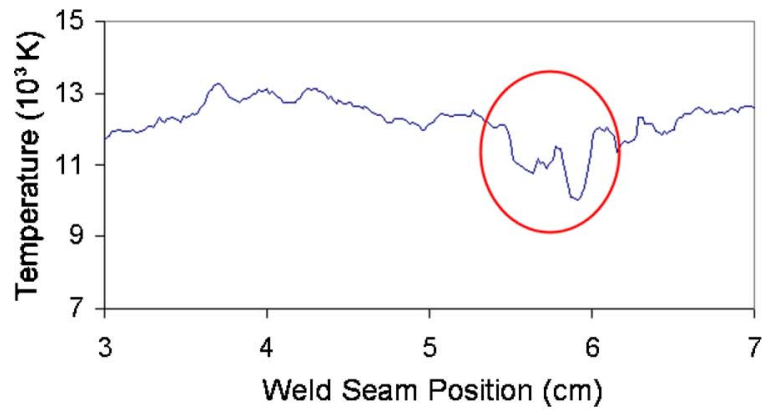

c) Te calculated with pair of $\mathrm{Ar}$ II lines

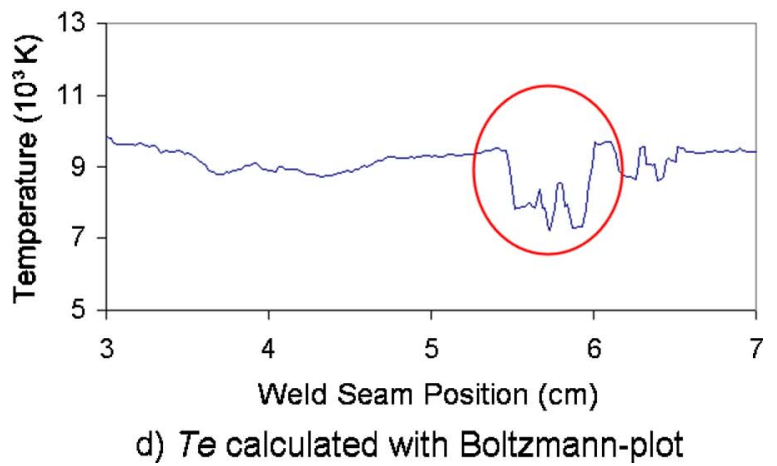

Fig. 6 Detection of gas cut.

creases, as can be observed in Fig. 4(d). It must be noted that not only the change in the $T_{e}$ profile is more pronounced when using the Boltzmann plot, but also the $T_{e}$ profile, when no defects are present, is clearly less noisy than the ones depicted in Figs. 4(b) and 4(c).

A different test is presented in Fig. 5. In Figs. 5(a) (top) and 5(b) (bottom), the resulting weld seam is shown. In this case, a plate with a thickness of $1 \mathrm{~mm}$ was used, and the reduction of the gas flux resulted in a crater in the weld seam at $x \approx 3.5 \mathrm{~cm}$. The welding intensity is also varied at $x=2.5 \mathrm{~cm}$ from 44 to $34 \mathrm{~A}$, whose influence in the weld seam is obvious in Fig. 5(b). Although this discontinuity in the weld seam is not clearly identified in Fig. 5(c), where $T_{e}$ has been calculated with a pair of Ar II emission lines (located at 473 and $485 \mathrm{~nm}$ ), the crater is detected. In Fig. 5(d), the Boltzmann plot with four Ar II lines (with wavelengths of $473,476,480$, and $485 \mathrm{~nm}$ ) is used to obtain $T_{e}$. In this case, both the variation of intensity and the crater can be distinguished, as the first peak at $x=2.5 \mathrm{~cm}$ is associated with the intensity change, the second at $x=3 \mathrm{~cm}$ with 
the increase in weld penetration due to the gas flux reduction, and the third one at $x=3.5 \mathrm{~cm}$ with the beginning of the crater.

As a final test, a 3-mm-thick plate was used. The analysis was performed using a constant intensity of $45 \mathrm{~A}$ and reducing the shielding gas flux from 12 to $2 \mathrm{~L} / \mathrm{min}$ at $x$ $\approx 5.5 \mathrm{~cm}$. In Figs. 6(a) and 6(b), the resulting weld seam is presented, where the defect caused by the gas flux reduction is obvious. In Fig. 6(b), the previously mentioned incision on the plate is clearly observed. In Fig. 6(c), where the lines of Ar II with wavelengths of 461 and 475 have been used, the defect at $x \approx 5.5 \mathrm{~cm}$ is clearly recognized. However, the $T_{e}$ obtained in Fig. 6(d) by using the Boltzmann plot with four Ar II lines (located at 461, 473, 476, and $480 \mathrm{~nm}$ ) allows an easier identification of the defects, as $T_{e}$ is more stable when no defects are to be found.

The previously described results from the welding tests confirm that the real-time implementation of the Bolztmann-plot technique offers a robust and fast calculation of the electronic temperature of the plasma, and with better sensitivity regarding defect detection than methods based on single pairs of spectral lines.

Further research is being conducted at present to collect the light with an optical fiber embedded in the welding torch, thus allowing a noninvasive monitoring system more appropriate for a production environment. Field tests will be also presented and discussed.

\section{Conclusions}

A new real-time implementation of the Boltzmann-plot technique for optical spectroscopic analysis of thermal plasmas, with applications in on-line quality monitoring of arc welding processes, is presented. Although the Boltzmann-plot technique is a well-known method to calculate the electronic temperature of a plasma generated in an arc welding procedure, it is commonly not used in online quality assessment systems due to its high computational cost. The classical approach is to obtain $T_{e}$ by using only a single pair of emission lines from the plasma, but by involving several different lines in the Boltzmann plot, a more precise estimation of $T_{e}$ is expected to be obtained, and hence, an easier discrimination of the defects appearing along the weld seam. The experimental results, based on
TIG welding of steel plates, confirm not only the feasibility of using the Boltzmann plot for the $T_{e}$ calculation process in a real-time system, but also that it offers an easier way to identify weld defects.

\section{Acknowledgments}

The authors thank the Spanish Government under the Ministry of Science and Technology for its support through the EOAMOP (TIC'2002-01259) research and development project.

\section{References}

1. L. Li, D. J. Brookfield, and W. M. Steen, "Plasma charge sensor for in-process, non-contact monitoring of the laser welding process," Meas. Sci. Technol. 7(4), 615-626 (1996).

2. H. P. Gu and W. W. Duley, "Resonant acoustic emission during laser welding of metals," J. Phys. D 29(3), 550-555 (1996).

3. B. Grabas, J. Dardthuret, and M. Laurent, "Observation of penetration with infrared thermography in laser welding," J. Phys. IV 4(C4), 139-142 (1994).

4. H. R. Griem, Principles of Plasma Spectroscopy, Cambridge University Press, Cambridge, UK (1997).

5. C. J. Kim, S. H. Baik, M. S. Kim, and C. M. Chung, "Auto-focus control and weld process monitoring of laser welding using chromatic filtering of thermal radiation," Laser Process. Mat. Industrial Appl. 2, 388-398 (1998).

6. J. Bruncko, F. Uherek, D. Chorvat, M. Michalka, and P. Fodrek, "Spectroscopic investigation of laser induced plasma in laser beam welding," Proc. SPIE 4644, 109-115 (2002).

7. F. M. Haran, D. P. Hand, C. Peteres, and J. D. C. Jones, "Focus control system for laser welding," Appl. Opt. 36, 5246-5251 (1997).

8. P. Sforza and D. de Blasiis, "On-line optical monitoring system for arc welding," NDT \& E Int. 35, 37-43 (2002).

9. A. Ancona, V. Spagnolo, P. Mario Lugara, and M. Ferrara, "Optical sensor for real-time monitoring of $\mathrm{CO}_{2}$ laser welding process," Appl. Opt. 40(33), 6019-6025 (2001).

10. M. Ferrara, A. Ancona, P. Mario Lugara, and M. Sibilano, "On-line quality monitoring of welding processes by means of plasma optical spectroscopy," Proc. SPIE 3888, 750-758 (2000).

11. J. A. Aguilera and C. Aragón, "Characterization of a laser-induced plasma by spatially resolved spectroscopy of neutral atom and ion emissions. Comparison of local and spatially integrated measurements," Spectrochim. Acta, Part B 60, 59 (2004).

12. J. Mirapeix, A. Cobo, O. Conde, M. Ángeles Quintela, and J. M. López-Higuera, "Spectroscopic analysis technique for arc-welding process control," Proc. SPIE 5948, 594824 (2006).

13. T. Zeh, H. Schweizer, A. Meixner, A. Purde, and A. W. Koch, "Enhancement of detection accuracy of fiber Bragg grating sensors," Proc. SPIE 5502, 540-543 (2004).

14. W. Lochte-Holtgreven, Plasma Diagnostics, North-Holland Publishing Company, Amsterdam (1968).

Biographies and photographs of authors not available. 